MATHEMATICS OF COMPUTATION

Volume 81, Number 278, April 2012, Pages 861-877

S $0025-5718(2011) 02549-4$

Article electronically published on October 18, 2011

\title{
ON THE CONVERGENCE RATES OF LEGENDRE APPROXIMATION
}

\author{
HAIYONG WANG AND SHUHUANG XIANG
}

\begin{abstract}
The problem of the rate of convergence of Legendre approximation is considered. We first establish the decay rates of the coefficients in the Legendre series expansion and then derive error bounds of the truncated Legendre series in the uniform norm. In addition, we consider Legendre approximation with interpolation. In particular, we are interested in the barycentric Lagrange formula at the Gauss-Legendre points. Explicit barycentric weights, in terms of Gauss-Legendre points and corresponding quadrature weights, are presented that allow a fast evaluation of the Legendre interpolation formula. Error estimates for Legendre interpolation polynomials are also given.
\end{abstract}

\section{INTRODUCTION}

The Chebyshev and Legendre polynomials are two important sequences of orthogonal polynomials which have been extensively investigated and applied in interpolation and approximation theory [8, 11, 25, numerical integration [10, 12, 33, special function theory [27, the solution of the second- and fourth-order elliptic equations [15, 29, 30, computational fluid dynamics, etc. They are not only powerful tools for the approximation of functions that are difficult to compute, but also essential ingredients of numerical integration and approximate solution of differential and integral equations. For example, the growing interest in spectral methods is largely due to their superior approximation properties as compared with other methods like finite difference methods, finite element methods, etc. In particular, the Chebyshev and Legendre spectral methods have excellent error properties in the approximation of a globally smooth function. Almost every spectral methods textbook introduces the theoretical and numerical aspects of Chebyshev and Legendre polynomials (see, for instance, [5, 7, 13, 19, 20, 21, 31, 36]).

The orthogonal polynomial expansion occurs in a wide range of practical problems and applications and plays an important role in many fields of mathematics and physics. Let $T_{n}(x)$ and $P_{n}(x)$ denote the Chebyshev polynomial of the first kind and the Legendre polynomial of degree $n$, respectively. If the function $f(x)$ satisfies a Dini-Lipschitz condition on $[-1,1]$, then it has the following uniformly

Received by the editor May 7, 2010 and, in revised form, February 17, 2011.

2010 Mathematics Subject Classification. Primary 65D05, 65D99, 41A25.

Key words and phrases. Legendre expansion, Chebyshev expansion, Bernstein ellipse, barycentric Lagrange interpolation.

This work was supported by the NSF of China (No. 11071260).

(C)2011 American Mathematical Society Reverts to public domain 28 years from publication 
convergent Chebyshev series expansion [8, p. 129]

$$
f(x)=\sum_{n=0}^{\infty}{ }^{\prime} c_{n} T_{n}(x)
$$

where the prime denotes a summation whose first term is halved. The Chebyshev coefficients $c_{n}$ are given by

$$
c_{n}=\frac{2}{\pi} \int_{-1}^{1} \frac{f(x) T_{n}(x)}{\sqrt{1-x^{2}}} d x, \quad n=0,1, \ldots .
$$

It is well known that the computation of the Chebyshev coefficients can be carried out by the fast Fourier transform (FFT); hence Chebyshev expansions are widely used in numerical integration [9], approximation theory [8] and so on. If the function $f(x)$ belongs to a Lipschitz class of order greater than or equal to $1 / 2$ on $[-1,1]$, then it has the following uniformly convergent Legendre series expansion [32,

$$
f(x)=\sum_{n=0}^{\infty} a_{n} P_{n}(x),
$$

where

$$
a_{n}=\left(n+\frac{1}{2}\right) \int_{-1}^{1} f(x) P_{n}(x) d x, \quad n=0,1, \ldots
$$

The Legendre series expansion (1.3) is extensively applied in spherical harmonics, signal processing, control systems, the computation of the spectra of highly oscillatory Fredholm integral operators [6], the inverse polynomial reconstruction method for the resolution of the Gibbs phenomenon [24] and so on. In the last few years there has been an increasing interest in the computation of coefficients in the Legendre series expansion and several efficient algorithms have been developed. For example, Piessens [26] proposed to substitute the Chebyshev expansion (1.1) into the Legendre coefficients (1.4). Alpert and Rokhlin [3] have developed a fast algorithm for converting a Chebyshev series to a Legendre series or vice versa. Based on a hypergeometric transformation, Iserles [23 proposed a fast algorithm to compute the Legendre coefficients by using the FFT if the function $f$ is analytic in a neighborhood of the interval $[-1,1]$. These algorithms are efficient and cost $O\left(N \log _{2} N\right)$ operations for the computation of the first $N$ Legendre coefficients. We refer the reader to [3, 23, 26] for more details.

In this paper, we shall devote our attention to the convergence rate of Legendre approximation schemes, including the truncated series expansion and polynomial interpolation approximation. For an excellent and comprehensive treatment of the rate of convergence of Chebyshev approximation, we refer the reader to [35. We commence our analysis by establishing the decay rates of the coefficients in the Legendre series expansion, and further, we derive error bounds of the truncated Legendre series expansion. Moreover, we consider the case of Legendre interpolation approximation, and especially we concentrate our attention on the barycentric form of the Legendre interpolation formula. In recent years, the barycentric Lagrange interpolation formula has attracted much attention owing to its stability and high efficiency. We show that the barycentric weights for Gauss-Legendre points can be expressed explicitly from the corresponding points and quadrature weights. Thus, if we have precomputed the Gauss-Legendre points and corresponding quadrature weights, then the barycentric Legendre formula can be evaluated in $O(n)$ operations. 
Indeed, recent research has shown that the computation of the Gauss-Legendre quadrature points and weights can be carried out in $O(n)$ operations (see [17]). Hence each evaluation of the barycentric Legendre formula can be carried out in $O(n)$ operations, and this is asymptotically the same work required to compute the barycentric Chebyshev formulas.

This paper is organized as follows. In the next section, we discuss the decay rates of the Legendre coefficients and derive error bounds of the truncated Legendre series expansion in the uniform norm. In Section 3 we explore the barycentric form of the Legendre interpolation formula. The explicit form of the barycentric weights for Gauss-Legendre points is presented, which provide a fast way for the implementation of the Legendre interpolation formula. Error estimates of the Legendre interpolation polynomial are also given.

\section{Decay Rates of Legendre Coefficients}

In this section we shall establish the decay rates of the Legendre coefficients and give error bounds of the truncated Legendre series expansion. For this purpose, let $\|\cdot\|_{T}$ be the Chebyshev-weighted seminorm defined by

$$
\|u\|_{T}=\int_{-1}^{1} \frac{\left|u^{\prime}(x)\right|}{\sqrt{1-x^{2}}} d x .
$$

Theorem 2.1. If $f, f^{\prime}, \ldots, f^{(k-1)}$ are absolutely continuous on $[-1,1]$ and $\left\|f^{(k)}\right\|_{T}$ $=V_{k}<\infty$ for some $k \geq 1$, then for each $n>k+1$,

$$
\left|a_{n}\right| \leq \frac{V_{k}}{\left(n-\frac{1}{2}\right)\left(n-\frac{3}{2}\right) \cdots\left(n-\frac{2 k-1}{2}\right)} \sqrt{\frac{\pi}{2(n-k-1)}} .
$$

If $f$ is analytic inside and on the Bernstein ellipse $\mathcal{E}_{\varrho}$ with foci \pm 1 and major semiaxis and minor semiaxis summing to $\varrho>1$, then for each $n \geq 0$,

$$
\left|a_{n}\right| \leq \frac{(2 n+1) \ell\left(\mathcal{E}_{\varrho}\right) M}{\pi \varrho^{n+1}\left(1-\varrho^{-2}\right)},
$$

where $M=\max _{z \in \mathcal{E}_{\varrho}}|f(z)|$ and $\ell\left(\mathcal{E}_{\varrho}\right)$ denotes the length of the circumference of $\mathcal{E}_{\varrho}$.

Proof. First let us consider the case when $f$ is analytic inside and on $\mathcal{E}_{\varrho}$. By Cauchy's integral formula, we can write

$$
\begin{aligned}
a_{n} & =\left(n+\frac{1}{2}\right) \int_{-1}^{1} f(x) P_{n}(x) d x \\
& =\left(n+\frac{1}{2}\right) \int_{-1}^{1}\left(\frac{1}{2 \pi i} \oint_{\mathcal{E}_{\varrho}} \frac{f(z)}{z-x} d z\right) P_{n}(x) d x \\
& =\left(n+\frac{1}{2}\right) \frac{1}{\pi i} \oint_{\mathcal{E}_{\varrho}} f(z) Q_{n}(z) d z
\end{aligned}
$$

where

$$
Q_{n}(z)=\frac{1}{2} \int_{-1}^{1} \frac{P_{n}(x)}{z-x} d x, \quad n=0,1, \ldots,
$$

is the Legendre function of the second kind [1, p. 335] and $\mathcal{E}_{\varrho}$ denotes the Bernstein ellipse in the complex plane

$$
\mathcal{E}_{\varrho}=\left\{z \in \mathbb{C} \mid z=\frac{1}{2}\left(u+u^{-1}\right), u=\varrho e^{i \theta},-\pi \leq \theta \leq \pi\right\} .
$$


From Davis [11, p.311], we have

$$
Q_{n}(z)=\sum_{k=n+1}^{\infty} \frac{\sigma_{n, k}}{u^{k}(z)}, \quad n=0,1,2, \ldots
$$

where the coefficients $\sigma_{n, k}$ are given by

$$
\sigma_{n, k}=\int_{0}^{\pi} P_{n}(\cos t) \sin k t d t=\int_{-1}^{1} P_{n}(t) U_{k-1}(t) d t, \quad k \geq n+1,
$$

and $U_{k}(t)$ is the Chebyshev polynomial of the second kind of degree $k$. By noting the parity of the Legendre and Chebyshev polynomials, we have

$$
\sigma_{n, n+2 k}=0, \quad k \geq 1 \text {. }
$$

Combining equations (4.9.7), (4.9.8), (4.9.9) and (4.9.2) in 34, we have

$$
\sigma_{n, n+2 k-1}=\frac{2^{2 n-2 k+3}(n !)^{2}(n+1)_{k-1}(2 k-2) !}{(2 n+1) !((k-1) !)^{2}\left(n+\frac{3}{2}\right)_{k-1}},
$$

where the Pochhammer symbol $(a)_{k}$ is defined as $(a)_{0}=1,(a)_{k}=(a)_{k-1}(a+k-1)$, $k \geq 1$. It is easy to obtain the following inequality from (2.6),

$$
\left|\sigma_{n, n+2 k-1}\right| \leq 2, \quad n \geq 0, \quad k \geq 1 \text {. }
$$

Substituting (2.4) and (2.5) into (2.3) yields

$$
\begin{aligned}
a_{n} & =\left(n+\frac{1}{2}\right) \frac{1}{\pi i} \oint_{\mathcal{E}_{\varrho}} f(z) Q_{n}(z) d z \\
& =\left(n+\frac{1}{2}\right) \frac{1}{\pi i} \sum_{\substack{\infty=n+1 \\
\infty}} \sigma_{n, k} \oint_{\mathcal{E}_{\varrho}} \frac{f(z)}{u(z)^{k}} d z \\
& =\left(n+\frac{1}{2}\right) \frac{1}{\pi i} \sum_{j=1}^{\infty} \sigma_{n, n+2 j-1} \oint_{\mathcal{E}_{\varrho}} \frac{f(z)}{u(z)^{n+2 j-1}} d z .
\end{aligned}
$$

By noting the inequality (2.7), the Legendre coefficients $a_{n}$ can thus be bounded by

$$
\begin{aligned}
\left|a_{n}\right| & \leq\left(n+\frac{1}{2}\right) \frac{1}{\pi} \sum_{j=1}^{\infty}\left|\sigma_{n, n+2 j-1}\right| \oint_{\mathcal{E}_{\varrho}}\left|\frac{f(z)}{u(z)^{n+2 j-1}}\right||d z| \\
& \leq \frac{(2 n+1)}{\pi} \ell\left(\mathcal{E}_{\varrho}\right) M \sum_{j=1}^{\infty} \frac{1}{\varrho^{n+2 j-1}} \\
& =\frac{(2 n+1) \ell\left(\mathcal{E}_{\varrho}\right) M}{\pi \varrho^{n+1}\left(1-\varrho^{-2}\right)}
\end{aligned}
$$

This completes the proof of the inequality (2.2). 
As for (2.1), we proceed by using the following relation [21, p. 254]

$$
P_{n}(x)=\frac{1}{2 n+1}\left(P_{n+1}^{\prime}(x)-P_{n-1}^{\prime}(x)\right)
$$

and the fact that

$$
P_{n}( \pm 1)= \pm 1
$$

Applying integration by parts twice, we have

$$
\begin{aligned}
a_{n}= & \frac{1}{2}\left(\int_{-1}^{1} f(x) P_{n+1}^{\prime}(x) d x-\int_{-1}^{1} f(x) P_{n-1}^{\prime}(x) d x\right) \\
= & \frac{1}{2}\left(\left[f(x) P_{n+1}(x)-f(x) P_{n-1}(x)\right]_{-1}^{1}+\int_{-1}^{1} f^{\prime}(x) P_{n-1}(x) d x\right. \\
& \left.\quad-\int_{-1}^{1} f^{\prime}(x) P_{n+1}(x) d x\right) \\
= & \frac{1}{2}\left(\int_{-1}^{1} f^{\prime}(x) P_{n-1}(x) d x-\int_{-1}^{1} f^{\prime}(x) P_{n+1}(x) d x\right) \\
= & \frac{1}{2(2 n-1)} \int_{-1}^{1} f^{\prime}(x)\left(P_{n}^{\prime}(x)-P_{n-2}^{\prime}(x)\right) d x \\
& \quad-\frac{1}{2(2 n+3)} \int_{-1}^{1} f^{\prime}(x)\left(P_{n+2}^{\prime}(x)-P_{n}^{\prime}(x)\right) d x \\
= & \frac{1}{2 n-1} \int_{-1}^{1} f^{\prime \prime}(x)\left[\frac{P_{n-2}(x)}{2}-\frac{P_{n}(x)}{2}\right] d x \\
& \quad-\frac{1}{2 n+3} \int_{-1}^{1} f^{\prime \prime}(x)\left[\frac{P_{n}(x)}{2}-\frac{P_{n+2}(x)}{2}\right] d x,
\end{aligned}
$$

where the boundary terms vanish by noting (2.11). From Rainville [27, p. 173] we know

$$
\left|P_{n}(x)\right| \leq \sqrt{\frac{\pi}{2 n\left(1-x^{2}\right)}}, \quad-1<x<1
$$

which implies

$$
\begin{aligned}
\left|a_{n}\right| \leq & \frac{1}{2 n-1} \int_{-1}^{1} \frac{\left|f^{\prime \prime}(x)\right|}{\sqrt{1-x^{2}}}\left(\frac{1}{2} \sqrt{\frac{\pi}{2(n-2)}}+\frac{1}{2} \sqrt{\frac{\pi}{2 n}}\right) d x \\
& +\frac{1}{2 n+3} \int_{-1}^{1} \frac{\left|f^{\prime \prime}(x)\right|}{\sqrt{1-x^{2}}}\left(\frac{1}{2} \sqrt{\frac{\pi}{2 n}}+\frac{1}{2} \sqrt{\frac{\pi}{2(n+2)}}\right) d x \\
\leq & \frac{2}{2 n-1} \sqrt{\frac{\pi}{2(n-2)}} V_{1} \\
= & \frac{V_{1}}{n-\frac{1}{2}} \sqrt{\frac{\pi}{2(n-2)}}
\end{aligned}
$$


Thus we have proved the inequality (2.1) for the case $k=1$. Similarly, using the equation (2.10) and renewed integration by parts of (2.12), we have

$$
\begin{aligned}
a_{n}= & \frac{1}{2 n-1} \int_{-1}^{1} f^{\prime \prime}(x)\left[\frac{P_{n-1}^{\prime}(x)-P_{n-3}^{\prime}(x)}{2(2 n-3)}-\frac{P_{n+1}^{\prime}(x)-P_{n-1}^{\prime}(x)}{2(2 n+1)}\right] d x \\
& -\frac{1}{2 n+3} \int_{-1}^{1} f^{\prime \prime}(x)\left[\frac{P_{n+1}^{\prime}(x)-P_{n-1}^{\prime}(x)}{2(2 n+1)}-\frac{P_{n+3}^{\prime}(x)-P_{n+1}^{\prime}(x)}{2(2 n+5)}\right] d x \\
= & \frac{1}{(2 n-1)(2 n-3)} \int_{-1}^{1} f^{\prime \prime \prime}(x)\left[\frac{P_{n-3}(x)}{2}-\frac{P_{n-1}(x)}{2}\right] d x \\
& -\frac{1}{(2 n-1)(2 n+1)} \int_{-1}^{1} f^{\prime \prime \prime}(x)\left[\frac{P_{n-1}(x)}{2}-\frac{P_{n+1}(x)}{2}\right] d x \\
& -\frac{1}{(2 n+3)(2 n+1)} \int_{-1}^{1} f^{\prime \prime \prime}(x)\left[\frac{P_{n-1}(x)}{2}-\frac{P_{n+1}(x)}{2}\right] d x \\
& \frac{1}{(2 n+3)(2 n+5)} \int_{-1}^{1} f^{\prime \prime \prime}(x)\left[\frac{P_{n+1}(x)}{2}-\frac{P_{n+3}(x)}{2}\right] d x .
\end{aligned}
$$

By using (2.13) again, we get

$$
\begin{aligned}
\left|a_{n}\right| \leq & \frac{1}{(2 n-3)(2 n-1)} \int_{-1}^{1} \frac{\left|f^{\prime \prime \prime}(x)\right|}{\sqrt{1-x^{2}}}\left(\frac{1}{2} \sqrt{\frac{\pi}{2(n-3)}}+\frac{1}{2} \sqrt{\frac{\pi}{2(n-1)}}\right) d x \\
& +\frac{1}{(2 n-1)(2 n+1)} \int_{-1}^{1} \frac{\left|f^{\prime \prime \prime}(x)\right|}{\sqrt{1-x^{2}}}\left(\frac{1}{2} \sqrt{\frac{\pi}{2(n-1)}}+\frac{1}{2} \sqrt{\frac{\pi}{2(n+1)}}\right) d x \\
& +\frac{1}{(2 n+1)(2 n+3)} \int_{-1}^{1} \frac{\left|f^{\prime \prime \prime}(x)\right|}{\sqrt{1-x^{2}}}\left(\frac{1}{2} \sqrt{\frac{\pi}{2(n-1)}}+\frac{1}{2} \sqrt{\frac{\pi}{2(n+1)}}\right) d x \\
& +\frac{1}{(2 n+3)(2 n+5)} \int_{-1}^{1} \frac{\left|f^{\prime \prime \prime}(x)\right|}{\sqrt{1-x^{2}}}\left(\frac{1}{2} \sqrt{\frac{\pi}{2(n+1)}}+\frac{1}{2} \sqrt{\frac{\pi}{2(n+3)}}\right) d x \\
\leq & \frac{4}{(2 n-3)(2 n-1)} \sqrt{\frac{\pi}{2(n-3)}} V_{2} \\
= & \frac{V_{2}}{\left(n-\frac{1}{2}\right)\left(n-\frac{3}{2}\right)} \sqrt{\frac{\pi}{2(n-3)}} .
\end{aligned}
$$

The case $k=2$ is proved. Repeated applications of integration by parts and (2.10), (2.11), (2.13) bring in higher derivatives of $f$ and corresponding higher variations up to $V_{k}$. Hence we can obtain the desired result (2.1) but we omit the details.

Remark 2.2. If $f$ is analytic inside and on $\mathcal{E}_{\varrho}$, the estimate for the Legendre coefficients is also given by [11, p. 313]

$$
\left|a_{n}\right| \leq \frac{(2 n+1) \ell\left(\mathcal{E}_{\varrho}\right) M}{2 \varrho^{n+1}\left(1-\varrho^{-1}\right)}, \quad n \geq 0 .
$$

Comparing inequalities (2.2) and (2.17) we find that the ratio of the former to the latter is equal to $\frac{2\left(1-\varrho^{-1}\right)}{\pi\left(1-\varrho^{-2}\right)}$. Thus the ratio is less than one for $\varrho>1$ and the former is better. 

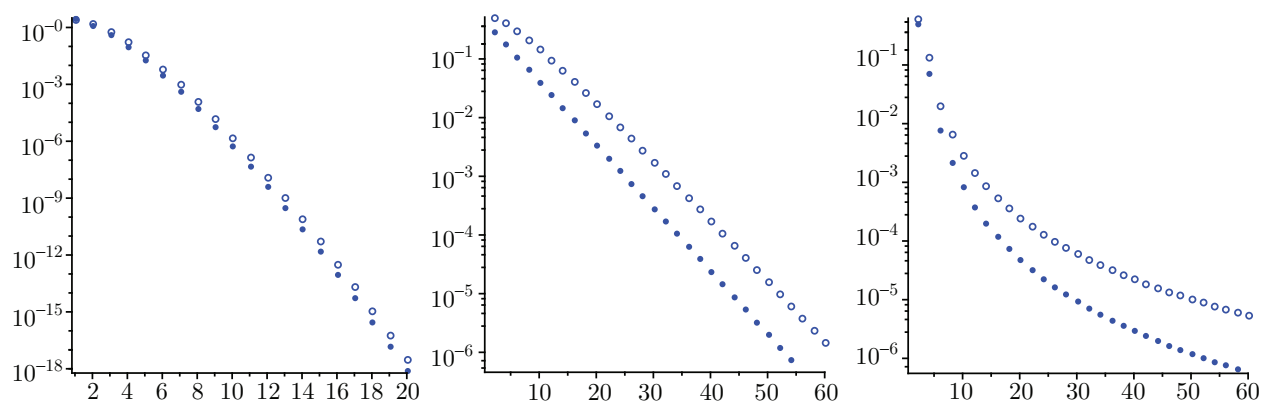

Figure 1. The absolute value of the $n$-th Chebyshev coefficient (dots) and the $n$-th Legendre coefficient (circles) for the three functions $e^{2 x}$ (left), $\frac{1}{1+16 x^{2}}$ (middle) and $|x|^{3}$ (right).

Remark 2.3. Under the same assumption as in Remark 2.2, substituting $z=\frac{1}{2}(u+$ $\left.u^{-1}\right)$ into (2.8) yields

$$
\begin{aligned}
a_{n}= & \left(n+\frac{1}{2}\right) \frac{1}{\pi i} \sum_{j=1}^{\infty} \sigma_{n, n+2 j-1} \oint_{\mathcal{E}_{\varrho}} \frac{f(z)}{u(z)^{n+2 j-1}} d z \\
= & \left(n+\frac{1}{2}\right) \frac{1}{2 \pi} \sum_{j=1}^{\infty} \sigma_{n, n+2 j-1} \int_{-\pi}^{\pi} f\left(\frac{1}{2}\left(\varrho e^{i \theta}+\varrho^{-1} e^{-i \theta}\right)\right) \\
& \times\left(1-\varrho^{-2} e^{-2 i \theta}\right)\left(\varrho e^{i \theta}\right)^{-n-2 j+2} d \theta .
\end{aligned}
$$

Here the integrands on the right side of the last equality are periodic functions of $\theta$ with period $2 \pi$. Hence the integrals can be computed efficiently by FFT. Thus we have provided an alternative simple derivation of Iserles' algorithm [23].

In [35] Trefethen has given the decay rates of the Chebyshev coefficients.

Lemma 2.4. If $f, f^{\prime}, \ldots, f^{(k-1)}$ are absolutely continuous on $[-1,1]$ and $\left\|f^{(k)}\right\|=$ $V_{k}<\infty$ for some $k \geq 1$, then for each $n \geq k+1$,

$$
\left|c_{n}\right| \leq \frac{2 V_{k}}{\pi n(n-1) \cdots(n-k)} .
$$

If $f$ is analytic inside and on $\mathcal{E}_{\varrho}$, then for each $n \geq 0$,

$$
\left|c_{n}\right| \leq \frac{2 M}{\varrho^{n}},
$$

where $M$ is as in (2.2).

Comparing the decay rates of the Legendre and Chebyshev coefficients, one can see that the estimates of the Chebyshev coefficients are smaller than those of Legendre coefficients. If $f$ is analytic in a neighborhood of the interval $[-1,1]$, then both the Legendre and Chebyshev coefficients decay exponentially as can be seen from (2.2) and (2.19). Figure 1 illustrates the decay rates of the two kinds of coefficients for the three functions $e^{2 x}, \frac{1}{1+16 x^{2}},|x|^{3}$. The first test function is analytic in the whole complex plane. As we can see, both coefficients decay exponentially and the Chebyshev coefficients show a slightly faster decay than their Legendre counterparts. The second test function is the Runge function which is analytic except at the two poles $\pm \frac{1}{4} i$. The last test function is not analytic in 

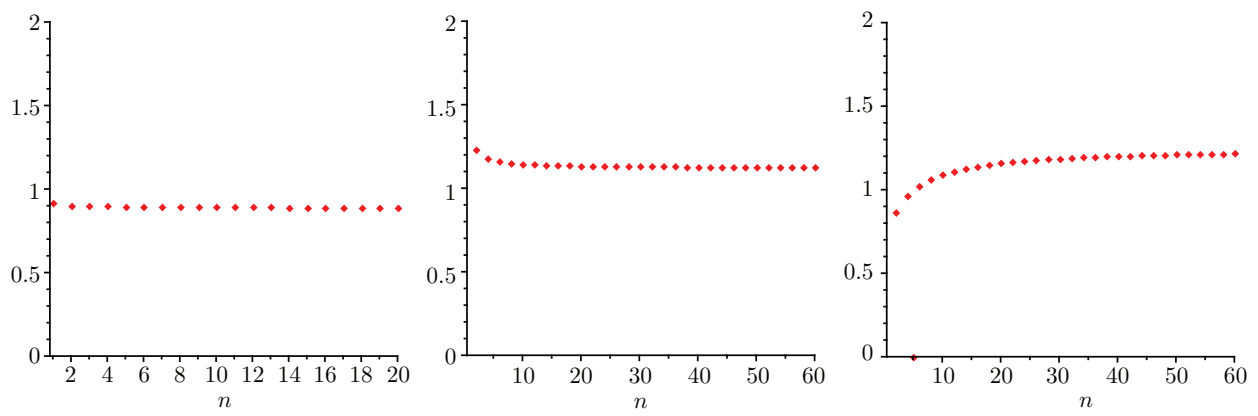

Figure 2. The ratio of the absolute value of the $n$-th Chebyshev coefficient $c_{n}$ to the $n$-th Legendre coefficient $a_{n}$ scaled by $\sqrt{n}$ for the three functions $e^{2 x}$ (left), $\frac{1}{1+16 x^{2}}$ (middle) and $|x|^{3}$ (right).

the complex plane. Obviously, the decay rates of the Chebyshev coefficients of the last two functions are always faster than those of Legendre coefficients. Figure 2 illustrates the ratio of the absolute value of the $n$-th Chebyshev coefficients $c_{n}$ to the $n$-th Legendre coefficient $a_{n}$ scaled by $\sqrt{n}$ for the above three test functions. In fact, Fox and Parker have compared both coefficients and asserted that the Chebyshev coefficients decay a factor of $\sqrt{n}$ faster than their Legendre counterparts [14, p. 17].

With the help of the decay rates of the Legendre coefficients, we next consider the truncated Legendre series. Let $f_{n}(x)$ denote the truncated Legendre series expansion of $f(x)$,

$$
f_{n}(x)=\sum_{j=0}^{n} a_{j} P_{j}(x)
$$

The following theorem is an immediate consequence of Theorem 2.1.

Theorem 2.5. If $f, f^{\prime}, \ldots, f^{(k-1)}$ are absolutely continuous on $[-1,1]$ and $\left\|f^{(k)}\right\|_{T}=V_{k}<\infty$ for some $k>1$, then for each $n>k+1$,

$$
\left|f(x)-f_{n}(x)\right| \leq \frac{V_{k}}{(k-1)\left(n-\frac{1}{2}\right)\left(n-\frac{3}{2}\right) \cdots\left(n-\frac{2 k-3}{2}\right)} \sqrt{\frac{\pi}{2(n-k)}}, \quad x \in[-1,1] .
$$

If $f$ is analytic inside and on $\mathcal{E}_{\varrho}$, then for each $n \geq 0$,

$$
\left|f(x)-f_{n}(x)\right| \leq \frac{(2 n \varrho+3 \varrho-2 n-1) \ell\left(\mathcal{E}_{\varrho}\right) M}{\pi \varrho^{n+1}(\varrho-1)^{2}\left(1-\varrho^{-2}\right)}, \quad x \in[-1,1] .
$$

Proof. The Legendre polynomials can be bounded by one, that is,

$$
\left|P_{n}(x)\right| \leq 1,
$$

where $x \in[-1,1]$ and $n \geq 0$. By virtue of the uniform convergence of the Legendre series expansion (1.3), we can subtract the truncated Legendre expansion from the infinite series and obtain

$$
\left|f(x)-f_{n}(x)\right|=\left|\sum_{j=n+1}^{\infty} a_{j} P_{j}(x)\right| \leq \sum_{j=n+1}^{\infty}\left|a_{j}\right| .
$$


The inequality (2.20) follows from (2.22) and (2.1),

$$
\begin{aligned}
\left|f(x)-f_{n}(x)\right| & \leq \sum_{j=n+1}^{\infty} \frac{V_{k}}{\left(j-\frac{1}{2}\right)\left(j-\frac{3}{2}\right) \cdots\left(j-\frac{2 k-1}{2}\right)} \sqrt{\frac{\pi}{2(j-k-1)}} \\
& \leq V_{k} \sqrt{\frac{\pi}{2(n-k)}} \sum_{j=n+1}^{\infty} \frac{1}{\left(j-\frac{1}{2}\right)\left(j-\frac{3}{2}\right) \cdots\left(j-\frac{2 k-1}{2}\right)} .
\end{aligned}
$$

Therefore, we have for $k>1$,

$$
\begin{aligned}
\mid f(x) & -f_{n}(x) \mid \leq V_{k} \sqrt{\frac{\pi}{2(n-k)}} \sum_{j=n+1}^{\infty} \frac{1}{\left(j-\frac{1}{2}\right)\left(j-\frac{3}{2}\right) \cdots\left(j-\frac{2 k-1}{2}\right)} \\
& =V_{k} \sqrt{\frac{\pi}{2(n-k)}} \frac{1}{k-1} \sum_{j=n+1}^{\infty}\left[\frac{1}{\left(j-\frac{3}{2}\right) \cdots\left(j-\frac{2 k-1}{2}\right)}-\frac{1}{\left(j-\frac{1}{2}\right) \cdots\left(j-\frac{2 k-3}{2}\right)}\right] \\
& =\frac{V_{k}}{(k-1)\left(n-\frac{1}{2}\right)\left(n-\frac{3}{2}\right) \cdots\left(n-\frac{2 k-3}{2}\right)} \sqrt{\frac{\pi}{2(n-k)}} .
\end{aligned}
$$

Similarly, (2.21) follows from (2.22) and (2.2),

$$
\begin{aligned}
\left|f(x)-f_{n}(x)\right| & \leq \sum_{j=n+1}^{\infty} \frac{(2 j+1) \ell\left(\mathcal{E}_{\varrho}\right) M}{\pi \varrho^{j+1}\left(1-\varrho^{-2}\right)} \\
& =\frac{\ell\left(\mathcal{E}_{\varrho}\right) M}{\pi\left(1-\varrho^{-2}\right)} \sum_{j=n+1}^{\infty} \frac{2 j+1}{\varrho^{j+1}} \\
& =\frac{(2 n \varrho+3 \varrho-2 n-1) \ell\left(\mathcal{E}_{\varrho}\right) M}{\pi \varrho^{n+1}(\varrho-1)^{2}\left(1-\varrho^{-2}\right)} .
\end{aligned}
$$

Hence we obtain the desired results and the proof is complete.

\section{BARYCENTRIC LEGENDRE FORMULA}

In this section, we turn our attention to the problem of Legendre interpolation approximation and present a fast algorithm for the efficient computation of the interpolation formula using Gauss-Legendre points. Interpolation is one of the most important tools of numerical analysis and almost every numerical analysis textbook has a chapter to introduce it (see, for instance, 2, 10, 16, 33]). The Lagrange interpolation polynomial $q_{n}(x)$ which interpolates the function $f(x)$ at the $(n+1)$ points $x_{j}$ may be written as

$$
q_{n}(x)=\sum_{j=0}^{n} f\left(x_{j}\right) \ell_{j}(x),
$$

where

$$
\ell_{j}(x)=\prod_{k=0, k \neq j}^{n} \frac{x-x_{k}}{x_{j}-x_{k}}, \quad j=0,1, \ldots, n,
$$

are the Lagrange fundamental polynomials. The Lagrange form (3.1) is praised for analytic utility and beauty but deplored for numerical practice [2]. In particular, the computation of (3.1) is numerically unstable and requires $O\left(n^{2}\right)$ operations, and every time a node $x_{j}$ is modified or added, all Lagrange fundamental polynomials have to be recalculated. 
The barycentric formula, a variant of Lagrange interpolation, has received considerable attention due to its attractive features such as low cost, stability and high efficiency. The barycentric Lagrange interpolation formula has the form

$$
q_{n}(x)=\frac{\sum_{j=0}^{n} \frac{\lambda_{j}}{x-x_{j}} f\left(x_{j}\right)}{\sum_{j=0}^{n} \frac{\lambda_{j}}{x-x_{j}}},
$$

where the barycentric weights $\lambda_{j}$ are defined by

$$
\lambda_{j}=\frac{1}{\prod_{k \neq j}\left(x_{j}-x_{k}\right)}, \quad j=0,1, \ldots, n .
$$

The barycentric formula (3.2) has a beautiful symmetric form and is eminently suitable for machine computation [10, p. 370]. For certain particular sets of points, such as equidistant points as well as Chebyshev points, the barycentric weights $\lambda_{j}$ can be computed analytically. For example, for Chebyshev points of the second kind

$$
x_{j}=\cos \frac{j \pi}{n}, \quad j=0,1, \ldots, n,
$$

the barycentric weights are given up to a common factor by [4, 28,

$$
\lambda_{j}^{\mathrm{CH} 2}=(-1)^{j} \delta_{j}, \quad \delta_{j}=\left\{\begin{array}{cc}
1 / 2, & j=0 \text { or } j=n, \\
1, & \text { otherwise }
\end{array}\right.
$$

For the barycentric weights of equidistant points and Chebyshev points of the first kind, we refer the reader to 4. With them each evaluation of the barycentric Lagrange formula $q_{n}(x)$ for equidistant or Chebyshev points requires only $O(n)$ operations. Moreover, Higham has shown in 22 that the barycentric Lagrange formula (3.2) is stable for any set of interpolating points with a small Lebesgue constant, such as Chebyshev and Gauss-Legendre points, and ought to be the standard method of polynomial interpolation 4. For an exhaustive discussion of the theoretical advantage of the barycentric Lagrange formula, we refer the reader to 4, 10, 16, 22.

If $f(x)$ is continuous and of bounded variation on $[-1,1]$, then the Lagrange interpolation polynomial $q_{n}(x)$ which interpolates $f(x)$ at the Gauss-Legendre points converges uniformly to $f(x)$ on $[-1,1]$ 38. The calculation of the barycentric interpolation formula for Gauss-Legendre points, that is, when the $x_{j}$ are zeros of $P_{n+1}(x)$, has not been investigated as extensively in the literature as that of the Chebyshev cases. Indeed, Berrut and Trefethen in [4 omitted the discussion of the Legendre case and just mentioned that explicit barycentric weights $\lambda_{j}$ are not known for Gauss-Legendre points. In the following we shall derive the barycentric weights for Gauss-Legendre points. For convenience, we assume throughout this paper that the Gauss-Legendre points $x_{j}$ are monotonic.

Theorem 3.1. For Gauss-Legendre points, the barycentric weights are given by

$$
\lambda_{j}^{G L}=(-1)^{j} \sqrt{\left(1-x_{j}^{2}\right) w_{j}}, \quad j=0, \ldots, n,
$$

where $w_{j}$ are the weights in the Gauss-Legendre quadrature formula. 
Proof. For Gauss-Legendre points, the Gauss quadrature weights are given by [7, p. 76]

$$
w_{j}=\frac{2}{\left(1-x_{j}^{2}\right)\left[P_{n+1}^{\prime}\left(x_{j}\right)\right]^{2}} .
$$

From equation (3.3), we have that the barycentric weights for Gauss-Legendre points can be written as

$$
\lambda_{j}=\frac{1}{\prod_{k \neq j}\left(x_{j}-x_{k}\right)}=\frac{d_{n+1}}{P_{n+1}^{\prime}\left(x_{j}\right)},
$$

where $d_{n+1}$ is the leading coefficient of $x^{n+1}$ in $P_{n+1}(x)$. Substituting (3.7) into (3.6), we have

$$
w_{j}=\frac{2 \lambda_{j}^{2}}{d_{n+1}^{2}\left(1-x_{j}^{2}\right)}
$$

Consequently,

$$
\lambda_{j}^{2}=d_{n+1}^{2} \frac{w_{j}\left(1-x_{j}^{2}\right)}{2} .
$$

From equation (3.3) it is not difficult to show that the barycentric weights $\lambda_{j}$ have alternating signs. Thus,

$$
\lambda_{j}=\sigma(-1)^{j} \sqrt{\frac{d_{n+1}^{2} w_{j}\left(1-x_{j}^{2}\right)}{2}},
$$

where $\sigma= \pm 1$. Cancelling the common factors independent of $j$ yields

$$
\lambda_{j}^{G L}=(-1)^{j} \sqrt{\left(1-x_{j}^{2}\right) w_{j}} .
$$

This completes the proof of Theorem 3.1.

Remark 3.2. Equation (3.5) shows a close connection between the barycentric weights and the Gauss-Legendre quadrature points and weights. This idea can be further extended to the Jacobi case. We shall show that for Gauss-Jacobi points, the barycentric weights can also be expressed in terms of Gauss-Jacobi quadrature points and weights [39].

The computation of the Gauss-Legendre points $x_{j}$ and corresponding quadrature weights $w_{j}$ has been extensively investigated in the past decades. For quite some time, it has been best to consider a symmetric tridiagonal matrix whose elements are obtained from the three-term recurrence relation satisfied by the Legendre polynomials [18; this algorithm for the $x_{j}$ and $w_{j}$ costs $O\left(n^{2}\right)$ operations. Recently, a fast algorithm, which costs only $O(n)$ operations, has been developed by Glaser, Liu and Rokhlin for computing the Gauss-Legendre points and corresponding quadrature weights by using the Prüfer transform and the classical Taylor series method. We refer the reader to [17] for a deeper treatment. Therefore, the barycentric Legendre formula can be carried out in $O(n)$ operations and this is asymptotically the same required work for computing the barycentric Chebyshev formulas. Figure 3 illustrates the barycentric Lagrange interpolation of the function $f(x)=|\sin \pi x|-x$ in 21 (thus $n=20$ ) and 101 Gauss-Legendre points $(n=100)$ on $[-1,1]$. For the sake of simplicity, in this example we have precomputed the Gauss-Legendre points 

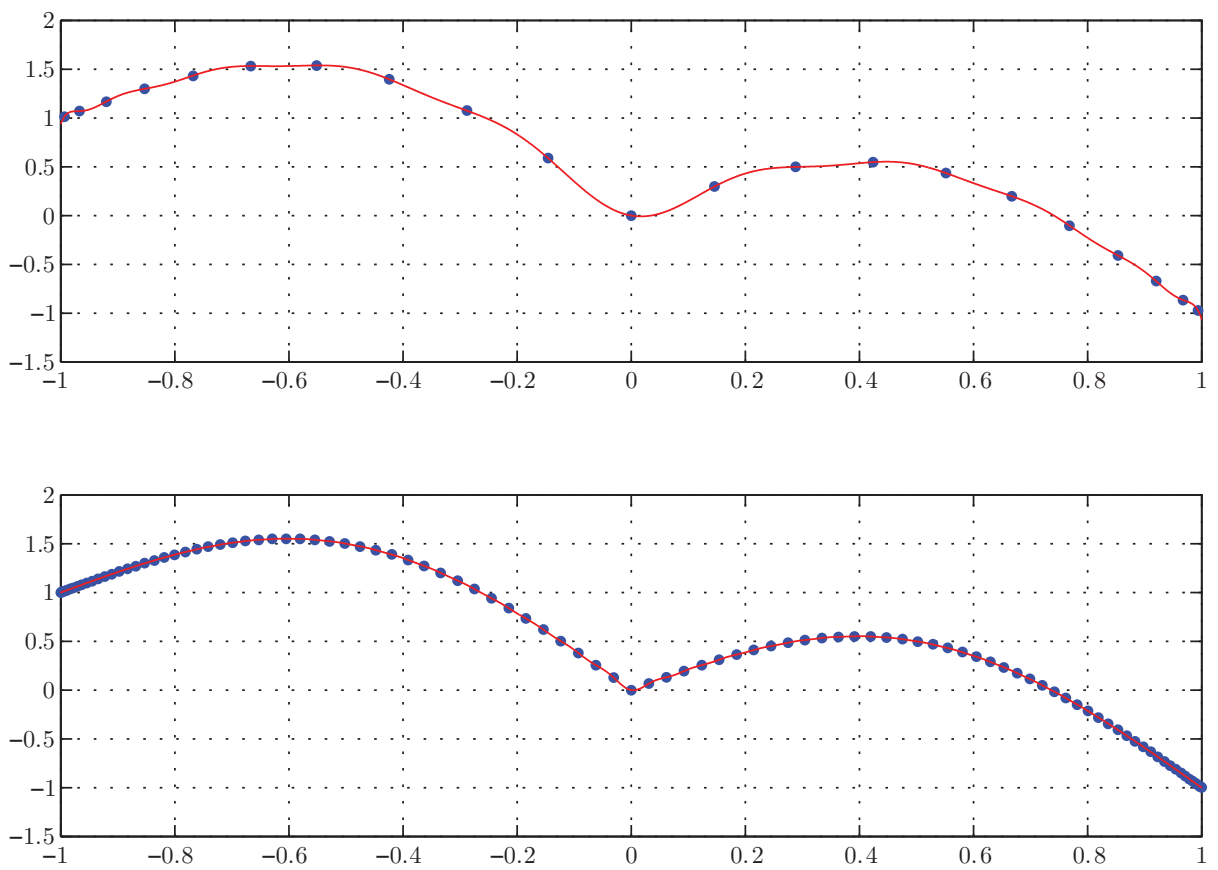

Figure 3. Barycentric Lagrange interpolation of the function $f(x)=|\sin \pi x|-x$ for $n=20$ (top) and $n=100$ (bottom). The dots denote the interpolated values $f\left(x_{j}\right)$.

$x_{j}$ and quadrature weights $w_{j}$ by using the MatLaB code in 35. and computed the barycentric weights $\lambda_{j}^{G L}$ by (3.5). We remark that, since the barycentric weights $\lambda_{j}^{G L}$ can be evaluated efficiently, the Legendre interpolation formula can be obtained in a fast way.

How good is the Legendre interpolation polynomial $q_{n}(x)$ ? Our starting point is the classical inequality [21, p. 100],

$$
\left|f(x)-q_{n}(x)\right| \leq\left(1+\Lambda_{n}\right)\left\|f(x)-p^{*}(x)\right\|_{\infty},
$$

where $p^{*}(x)$ is the best approximation of $f(x)$ by polynomials of degree $n$ or less, and

$$
\Lambda_{n}=\max _{-1 \leq x \leq 1} \sum_{j=0}^{n}\left|\ell_{j}(x)\right|
$$

is the Lebesgue constant for which the following estimate holds [13, p. 170]:

$$
\Lambda_{n}=O(\sqrt{n}) .
$$

It is well known that the truncated Chebyshev series expansion is very close to the best polynomial approximation [8, p. 126]. Hence if $f$ is analytic inside and on $\mathcal{E}_{\varrho}$, the error of the Legendre interpolation polynomial can be bounded approximately 
by

$$
\begin{aligned}
\left|f(x)-q_{n}(x)\right| & \leq\left(1+\Lambda_{n}\right)\left\|f(x)-\sum_{j=0}^{n}{ }^{\prime} c_{j} T_{j}(x)\right\|_{\infty} \\
& \leq\left(1+\Lambda_{n}\right) \frac{2 M}{\varrho^{n}(\varrho-1)} .
\end{aligned}
$$

Here we have made use of the Theorem 4.3 in $\left[35\right.$. By the same token, if $f, f^{\prime}, \ldots$, $f^{(k-1)}$ are absolutely continuous on $[-1,1]$ and $\left\|f^{(k)}\right\|_{T}=V_{k}<\infty$ for some $k \geq 1$, then for each $n \geq k+1$, by Lemma 2.4

$$
\begin{aligned}
\left|f(x)-q_{n}(x)\right| & \leq\left(1+\Lambda_{n}\right)\left\|f(x)-\sum_{j=0}^{n}{ }^{\prime} c_{j} T_{j}(x)\right\|_{\infty} \\
& \leq\left(1+\Lambda_{n}\right) \sum_{j=n+1}^{\infty} \frac{2 V_{k}}{\pi j(j-1) \cdots(j-k)} \\
& =\left(1+\Lambda_{n}\right) \frac{2 V_{k}}{k \pi n(n-1) \cdots(n-k+1)} .
\end{aligned}
$$

For error bounds of the Chebyshev interpolation formula, we refer the reader to 40.

We now give several numerical examples to demonstrate the convergence of the barycentric interpolation formula. For each $n$, the maximum error of the barycentric Legendre formula defined by

$$
\max _{x \in[-1,1]}\left|f(x)-q_{n}(x)\right|
$$

is measured at 10000 equispaced points in $[-1,1]$. The nodes and weights of the Gauss-Legendre quadrature rule are computed in the chebfun system by the command legpts 37. All computations in the following numerical examples have been performed in double precision arithmetic. Figure 4 shows the convergence of the barycentric interpolation formulas for Chebyshev points (3.4) and Gauss-Legendre points for the two analytic functions $f(x)=e^{x}$ and $f(x)=\cos (2 x+2)$. As we can see, the convergence of both formulas is exponential and is almost indistinguishable. Figure 5 illustrates the convergence of the barycentric formulas for the two
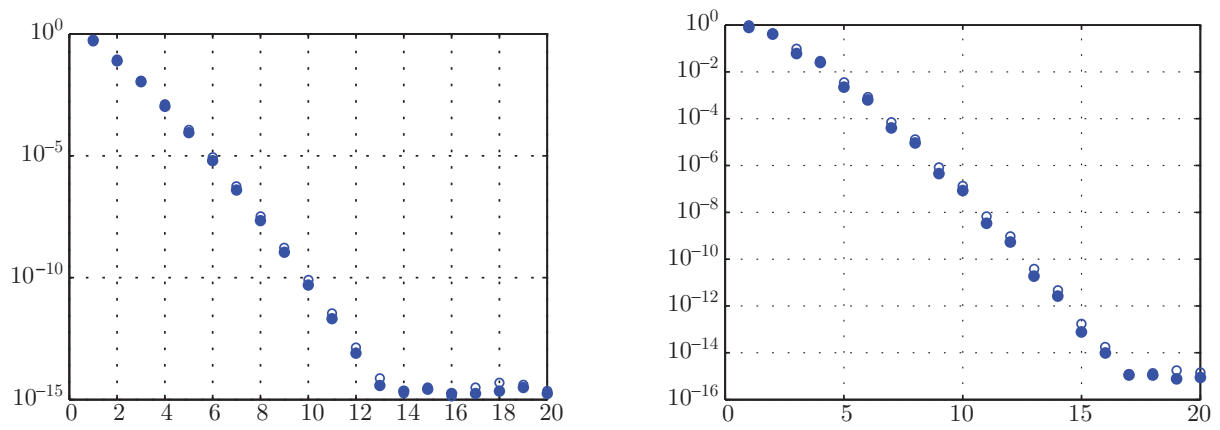

Figure 4. Convergence of the barycentric Chebyshev (dots) and Legendre (circles) interpolation formulas for the two functions $f(x)=e^{x}$ (left) and $f(x)=\cos (2 x+2)$ (right). 

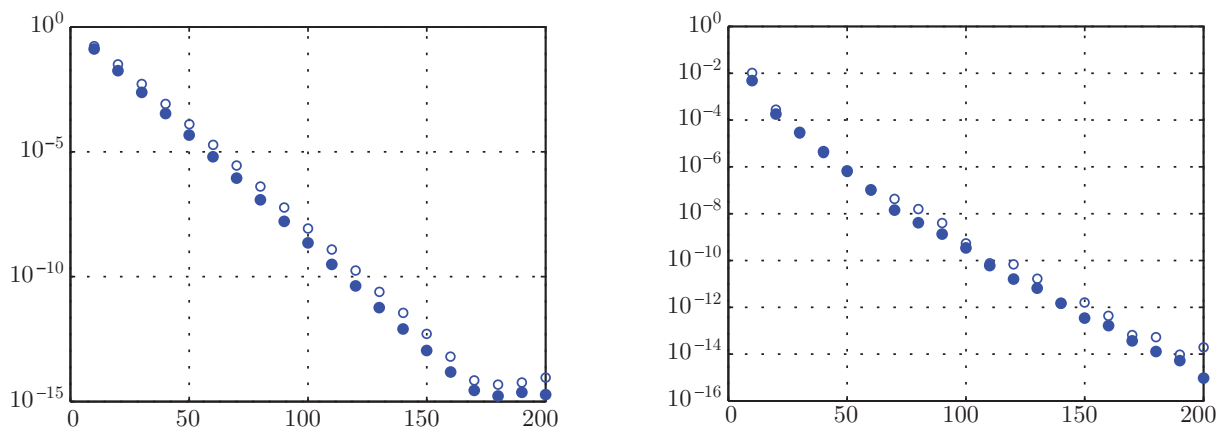

Figure 5. Convergence of barycentric Chebyshev (dots) and Legendre (circles) interpolation formulas for the functions $f(x)=$ $\frac{1}{1+25 x^{2}}$ (left) and $f(x)=e^{-1 / x^{2}}$ (right).
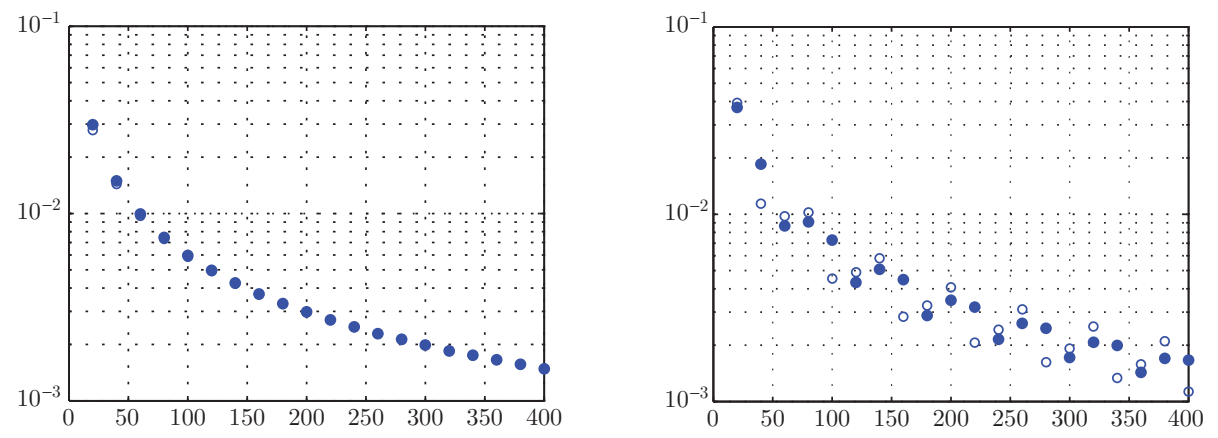

Figure 6. Convergence of barycentric Chebyshev (dots) and Legendre (circles) interpolation formulas for the functions $f(x)=|x|$ (left) and $f(x)=e^{|x+0.5|}$ (right).

functions $f(x)=\frac{1}{1+25 x^{2}}$ and $f(x)=e^{-1 / x^{2}}$ and Figure 6 illustrates the convergence of the barycentric formulas for the two non-analytic functions $f(x)=|x|$ and $f(x)=e^{|x+0.5|}$. As we can see, both formulas are of approximately equal accuracy.

Remark 3.3. For small as well as moderate values of $n$, the barycentric weights for Gauss-Legendre points can be computed stably and efficiently by using (3.5). For large values of $n$, however, equation (3.5) is unstable because the first and last Gauss-Legendre points are very close to \pm 1 . Therefore, the computation of (3.5) should be performed in extended precision at the moment.

In fact, the Gauss-Legendre and Chebyshev points (3.4) are distributed with the same density

$$
\frac{1}{\pi \sqrt{1-x^{2}}}
$$

that is, the grid points cluster quadratically near $x= \pm 1$. It is well known that grid points with this distribution are optimal in various senses for polynomial approximation and typically lead to well-behaved Lagrange interpolation [36, Chap. 5]. For a more detailed theoretical discussion of the accuracy of polynomial interpolation from the point of view of complex analysis, we refer the reader to [10, 21, 36]. 


\section{Concluding REMARKS}

In this paper, we have considered the decay rates of the Legendre coefficients. Based on this result, the error bounds of the truncated Legendre series expansion have been derived immediately. In addition, we considered the barycentric Legendre formula and showed that the barycentric weights for the Gauss-Legendre points can be expressed explicitly in terms of Gauss-Legendre points and corresponding quadrature weights. Thus, as in the Chebyshev cases, the barycentric Legendre interpolation formula can be carried out in a fast way. Numerical experiments indicate that the barycentric Legendre and Chebyshev formulas are of approximately equal accuracy.

In practice, both Chebyshev and Legendre interpolating polynomials are often used in numerical integration to derive quadrature rules. If the interpolating nodes are Gauss-Legendre or Chebyshev points of the second kind, the corresponding quadrature rules are Gauss-Legendre, respectively, Clenshaw-Curtis quadrature formulas. As shown by Trefethen in [35, Gauss-Legendre and Clenshaw-Curtis quadrature rules are of approximately equal accuracy for most integrands and both rules should be regarded as equally valuable and fundamental, with the former having an edge in elegance and the latter in simplicity 35 .

For many other applications of the barycentric interpolation formula, such as differentiation of polynomial interpolants, spectral methods for differential equations, estimation of the Lebesgue constant and fast multipole methods, we refer the reader to [4].

\section{ACKNOWLEDGEMENTS}

The first author acknowledges the financial support from the Hunan Provincial Innovation Foundation for Postgraduate. The authors are deeply grateful to Professor Nick Trefethen for his invaluable comments on the barycentric Legendre weights, and to two referees for detailed suggestions that have led to many improvements.

\section{REFERENCES}

1. M. Abramowitz and I. A. Stegun, Handbook of Mathematical Functions, National Bureau of Standards, Washington, D.C., 1964. MR0167642 (29:4914)

2. F. S. Acton, Numerical Methods that Usually Work, AMS, Providence, RI, 1990. MR 1074173 (92a:65002)

3. B. K. Alpert and V. Rokhlin, A fast algorithm for the evaluation of Legendre expansions, SIAM J. Sci. Statist. Comput., 12 (1991), 158-179. MR1078802(91i:65042)

4. J. P. Berrut and L. N. Trefethen, Barycentric Lagrange interpolation, SIAM Review, 46 (2004), 501-517. MR2115059 (2005k:65018)

5. J. P. Boyd, Chebyshev and Fourier Spectral Methods, Dover Publications, New York, 2000. MR.1874071 (2002k:65160)

6. H. Brunner, A. Iserles and S. P. Nørsett, The computation of the spectra of highly oscillatory Fredholm operators, J. Integral Equations Appl., to appear.

7. C. Canuto, M. Y. Hussaini, A. M. Quarteroni and T. A. Zang, Spectral methods: fundamentals in single domains, Springer, Berlin, 2006. MR2223552 (2007c:65001)

8. E. W. Cheney, Introduction to Approximation Theory, McGraw-Hill, New York, 1966. MR 0222517 (36:5568)

9. C. W. Clenshaw and A. R. Curtis, A method for numerical integration on an automatic computer, Numer. Math. 2 (1960), 197-205. MR 0117885 (22:8659)

10. G. Dahlquist and A. Björck, Numerical Methods in Scientific Computing, Volume I. SIAM, Philadelphia, 2007. MR2412832 (2009b:65003) 
11. P. J. Davis, Interpolation and Approximation, Dover Publications Inc., New York, 1975. MR0380189 (52:1089)

12. P. J. Davis and P. Rabinowitz, Methods of Numerical Integration, Second Edition, Academic Press, 1984. MR:760629 (86d:65004)

13. B. Fornberg, A Practical Guide to Pseudospectral Methods, Cambridge University Press, Cambridge, 1996. MR 1386891 (97g:65001)

14. L. Fox and I. B. Parker, Chebyshev Polynomials in Numerical Analysis, Oxford University Press, London, 1968. MR0228149 (37:3733)

15. D. Funaro, Spectral Elements for Transport-Dominated Equations, Springer, 1997. MR.1449871 (98g:65115)

16. W. Gautschi, Numerical Analysis: An Introduction, Birkhäuser, Boston, 1997. MR1454125 (98d:65001)

17. A. Glaser, X. Liu and V. Rokhlin, A fast algorithm for the calculation of the roots of special functions, SIAM J. Sci. Comput., 29 (2007), 1420-1438. MR2341794 (2009c:33056)

18. G. H. Golub and J. H. Welsch, Calculation of Gauss quadrature rules, Math. Comp., 23 (1969), 221-230. MR0245201 (39:6513)

19. D. Gottlieb and S. A. Orszag, Numerical Analysis of Spectral Methods: Theory and Applications, SIAM, Philadelphia, 1977. MR0520152(58:24983)

20. B. Y. Guo, Spectral Methods and Their Applications, World Scientific, Singapore, 1998. MR.1641586 (2000b:65194)

21. J. S. Hesthaven, S. Gottlieb and D. Gottlieb, Spectral Methods for Time-Dependent Problems, Cambridge University Press, 2007. MR 2333926 (2008i:65223)

22. N. J. Higham, The numerical stability of barycentric Lagrange interpolation, IMA J. Numer. Anal., 24 (2004), 547-556. MR.2094569(2005e:65007)

23. A. Iserles, A fast and simple algorithm for the computation of Legendre coefficients, Numer. Math., 217 (2011), 529-553.

24. J. H. Jung and B. D. Shizgal, On the numerical convergence with the inverse polynomial reconstruction method for the resolution of the Gibbs phenomenon, J. Comput. Phys., 224 (2007), 477-488. MR2330280 (2008h:42004)

25. J. C. Mason and D. C. Handscomb, Chebyshev Polynomials, CRC Press, New York, 2003. MR:1937591 (2004h:33001)

26. R. Piessens, Computation of Legendre series coefficients, Algorithm 473, Comm. ACM., 17 (1974), 25.

27. E. D. Rainville, Special Functions, Macmillan, New York, 1960. MR0107725 (21:6447)

28. H. E. Salzer, Lagrangian interpolation at the Chebyshev points $x_{n, \nu}=\cos (\nu \pi / n), \nu=0(1) n$; some unnoted advantages, Comput. J., 15 (1972), 156-159. MR0315865 (47:4414)

29. J. Shen, Efficient spectral-Galerkin method I. Direct solvers for the second and fourth order equations using Legendre polynomials, SIAM J. Sci. Comput., 15 (1994), 1489-1505. MR 1298626 (95j:65150)

30. J. Shen, Efficient spectral-Galerkin method II. direct solvers of second and fourth order equations by using Chebyshev polynomials, SIAM J. Sci. Comput., 16 (1995), 74-87. MR1311679 (95j:65151)

31. J. Shen and T. Tang, Spectral and High-Order Methods with Applications, Science Press, Beijing, 2006. MR2723481

32. P. K. Suetin, Representation of continuous and differentiable functions by Fourier series of Legendre polynomials, Soviet Math. Dokl., 5 (1964), 1408-1410.

33. E. Süli and D. Mayers, An Introduction to Numerical Analysis, Cambridge University Press, 2003. MR 2006500

34. G. Szegö, Orthogonal Polynomials, Colloquium Publications 23, American Mathematical Society, Providence, Rhode Island, 1939. MR0000077 (1:14b)

35. L. N. Trefethen, Is Gauss quadrature better than Clenshaw-Curtis?, SIAM Review., 50 (2008), 67-87. MR 2403058(2009c:65061)

36. L. N. Trefethen, Spectral Methods in MATLAB, SIAM, Philadelphia, 2000. MR1776072 (2001c:65001)

37. L. N. Trefethen, N. Hale, R. B. Platte, T. A. Driscoll and R. Pachón, Chebfun Version 3, http://www.maths.ox.ac.uk/chebfun/, University of Oxford, 2009.

38. P. Vértesi, Lagrange interpolation for continuous functions of bounded variation, Acta Math. Acad. Sci. Hungar., 35 (1980), 23-31. MR.588875 (82a:41007) 
39. H. Wang and D. Huybrechs, Explicit barycentric weights for polynomial interpolation in the roots of extrema of classical orthogonal polynomials, in preparation.

40. S. Xiang, X. Chen and H. Wang, Error bounds for approximation in Chebyshev points, Numer. Math., 116 (2010), 463-491. MR2684294

Department of Applied Mathematics and Software, Central South University, Changsha, Hunan 410083, People's Republic of China

Current address: Department of Computer Science, Katholieke Universiteit Leuven, Celestijnenlaan 200A, B-3001 Leuven, Belgium

E-mail address: haiyong.wang@cs.kuleuven.be

Department of Applied Mathematics and Software, Central South University, Changsha, Hunan 410083, People's Republic of China

E-mail address: xiangsh@mail.csu.edu.cn 